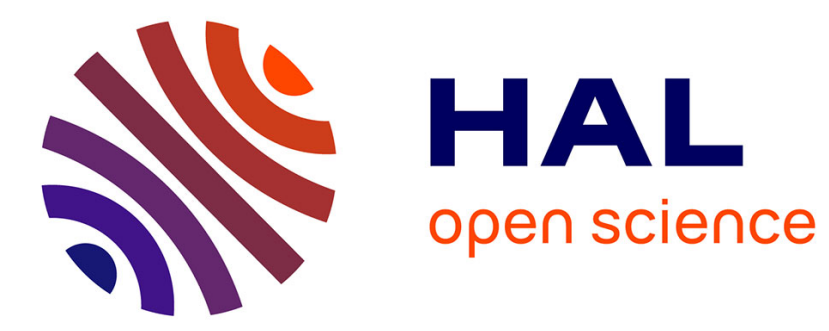

\title{
Critical piezoelectricity in percolation
}

Didier Sornette, Michel Lagier, Stéphane G. Roux, Alex Hansen

\section{To cite this version:}

Didier Sornette, Michel Lagier, Stéphane G. Roux, Alex Hansen. Critical piezoelectricity in percolation. Journal de Physique, 1989, 50 (16), pp.2201-2216. 10.1051/jphys:0198900500160220100 . jpa-00211055

\section{HAL Id: jpa-00211055 https://hal.science/jpa-00211055}

Submitted on 1 Jan 1989

HAL is a multi-disciplinary open access archive for the deposit and dissemination of scientific research documents, whether they are published or not. The documents may come from teaching and research institutions in France or abroad, or from public or private research centers.
L'archive ouverte pluridisciplinaire HAL, est destinée au dépôt et à la diffusion de documents scientifiques de niveau recherche, publiés ou non, émanant des établissements d'enseignement et de recherche français ou étrangers, des laboratoires publics ou privés. 
Classification

Physics Abstracts

$05.70 \mathrm{~J}-77.60$

\title{
Critical piezoelectricity in percolation
}

\author{
Didier Sornette $\left({ }^{1}\right)$, Michel Lagier $\left({ }^{2}\right)$, Stéphane Roux $\left({ }^{3}\right)$ and Alex Hansen $\left({ }^{4}\right)$
}

(') Laboratoire de Physique de la Matière Condensée, CNRS URA 190, Faculté des Sciences, Parc Valrose, 06034 Nice Cedex, France

$\left.{ }^{2}\right)$ Thomson-Sintra ASM, 525 route des Dolines, B.P. 38, Sophia-Antipolis, 06561 Valbonne, France

$\left({ }^{3}\right)$ Laboratoire de Physique de la Matière Hétérogène, CNRS URA 857, Ecole Supérieure de Physique et Chimie Industrielle, 10 rue Vauquelin, 75231 Paris Cedex 05, France

$\left({ }^{4}\right)$ IBM Bergen Scientific Center, Allégaten 36, N-5000 Bergen, Norway

(Reçu le 16 février 1989, accepté sous forme définitive le 3 mai 1989)

Résumé. - Nous étudions les propriétés piézoélectriques de céramiques très hétérogènes. L'effet piézoélectrique introduit un couplage entre les propriétés électriques et mécaniques, qui ont séparément des comportements d'échelle distincts. Son étude constitue donc une question a priori intéressante. Ce problème se pose de manière concrète dans le développement de céramiques piézoélectriques poreuses de très basse densité qui sont utilisées par exemple comme transducteurs électro-mécaniques. On considère un milieu piézoélectrique de constante diélectrique $\varepsilon_{\mathrm{r}}$ plongé dans un milieu de constante diélectrique constante unité ou proche de l'unité et de compliance très grande ou infinie. Deux cas intéressants sont étudiés : a) $\varepsilon_{\mathrm{r}} \gg 1$ and b) $\varepsilon_{\mathrm{r}} \approx 1$. Dans les deux cas, on trouve que le coefficient macroscopique de couplage piézoélectrique présente un comportement non-critique même quand les comportements mécanique (cas a) et b)) et électrique (dans le cas a)) exhibent leur comportement critique habituel. Cependant, nous prédisons l'existence de fluctuations critiques de la polarization sur le squelette du milieu piézoélectrique. De plus, le coefficient de couplage piézoélectrique en régime hydrostatique est fortement augmenté pour des matériaux très poreux proches du seuil de percolation ce qui est en accord avec les observations expérimentales. Nos arguments d'échelle sont comparés à des simulations numériques sur un modèle discret de "poutres" qui, on le sait, reproduit correctement les propriétés élastiques de systèmes dilués près de leur seuil de percolation.

Abstract. - We study the behaviour of piezoelectricity in highly heterogeneous media. The piezoelectric effect introduces a coupling between electrical and mechanical properties, which have separately different scaling behaviours. Its study therefore constitutes $a$ priori an interesting question. This problem is also suggested by the recent development of low-density, porous piezoelectric ceramics which are used, for example, as acoustic transducers. We consider a piezoelectric medium of dielectric constant $\varepsilon_{\mathrm{r}}$ plunged into a medium of dielectric constant unity or close to unity and large or infinite compliance. Two interesting cases are examined: a) $\varepsilon_{\mathrm{r}} \gg 1$ and b) $\varepsilon_{\mathrm{r}} \approx 1$. In both cases, we find that the spatial average of the piezoelectric coefficient is non-critical even if the mechanical (in cases a) and b)) or electrical behaviours (in case a)) do exhibit their usual critical behaviours near the percolation threshold. However, we predict the existence of critical fluctuations of the polarization of the piezoelectric backbone. Furthermore, 
the hydrostatic piezoelectric coefficient is found to increase strongly for highly porous systems, in agreement with observations. Our scaling arguments are supplemented by numerical computations on a discrete « beam » model, known to reproduce the critical elastic behaviour of diluted systems close to their percolation threshold.

\section{Introduction.}

The electrical and mechanical properties of composites have been intensively studied in the last few years with the achievement of a reasonable understanding for the whole range of concentrations or porosity. In particular, the use of the percolation model has allowed the prediction of the critical transport properties in the limit of large heterogeneity. Initially, the elastic properties of systems endowed with angular elasticity were thought to behave analogously to the electrical conductivity in percolation. It is now understood that the critical elastic behaviour is different (although related to), that of the conductivity [1].

The question of universality class of other models of randomly diluted medium without angular elasticity, the so-called central-force model [2], is still a debated issue, albeit some recent work [3] suggests that the critical elastic behavior of this model is similar to the case of usual percolation. Since at criticality the medium is still well connected, the electrical conductivity is not critical at the central-force percolation threshold.

However, in both kinds of models the mechanical and electrical properties of the systems close to their critical point, the percolation threshold, are different. In this work, we study the related problem of the behaviour of piezoelectricity in highly heterogeneous media. The introduction of a piezoelectric coupling between electrical and mechanical properties, which have separately different scaling behaviours, constitutes a priori an interesting question.

This problem is also suggested by the recent development of low-density, porous piezoelectric ceramics which are used, for example, as acoustic transducers [4-6]. A lowdensity piezoelectric should have better acoustic coupling with water and a more easily adjusted buoyancy than usual high-density PZT (lead zirconate-titanate) ceramics now used as hydrophones. The large compliance implies a large damping which is desirable in a passive device and a large deformability to any desired profile. Examples are provided by porous and ceramic-polymer structures. In porous ceramics, piezoelectric grains are sintered in the presence of a matrix which disappears after burning or washing. In this case, the space between grains is void. In ceramic-polymer structures, the space between grains is filled by a non-piezoelectric matrix of large compliance. The physical properties of these systems are just beginning to be explored experimentally : they seem to present a high sensitivity, a low loss allowing a large passing band as well as a high hydrostatic piezoelectric coefficient. Their modelling is the purpose of this paper.

Theoretically, many different experimental systems can be considered. We focus on the case of present practical interest where the piezoelectric material of dielectric constant $\varepsilon_{\mathrm{r}}$ is plunged into a medium of dielectric constant unity or close to unity and large or infinite compliance. Two interesting cases are examined : a) $\varepsilon_{\mathrm{r}} \gg 1$ and b) $\varepsilon_{\mathrm{r}} \approx 1$. In both cases, we find that the spatial average of the piezoelectric coefficient is non-critical even if the mechanical (in cases a) and b)) or electrical behaviours (in case a)) do exhibit their usual critical behaviours. However, we predict the existence of critical fluctuations of the polarization of the piezoelectric backbone. Furthermore, the hydrostatic piezoelectric coefficient is found to increase strongly for highly porous systems, in agreement with observations. Our arguments are supplemented by numerical computations on a discrete « beam » model [7], known to reproduce the critical elastic behaviour of diluted systems close to their percolation threshold. 


\section{Description of the model.}

2.1 Characteristics of THE POROUS PIEZoelectric CERAMic. - Piezoelectricity corresponds to the appearance of an electric polarization in a material submitted to a strain or stress (direct effect) or inversely to a deformation when the solid in submitted to an electric field (inverse effect). Piezoelectricity exists only in substances (crystals, ceramics...) having no center of symmetry [5]. As a result, a strain involves a different displacement of the positive and negative charges in the material resulting in the creation of small dipoles responsible for the appearance of a macroscopic electric polarization.

Ceramics are polycristalline systems made of microcrystallites having random orientations. As such, they should not qualify as piezoelectric due to their macroscopic isotropy coming from the random orientation of their microcrystallites : even though the individual crystals may be strongly piezoelectric, the ceramic is not so because the effects from the individual crystals cancel each other. However, ferroelectric ceramics can be rendered permanently anisotropic by applying an electric field : at first, each microcrystallite constitutes a small domain of non-zero ferroelectric spontaneous polarization with arbitrary orientation. The application of an external electric field orientates all the micro-domains in those directions allowed by the symmetry of each individual crystals which are nearest to that of the electric field. After this " poling » treatment, the ceramic resembles a single crystal which has a net dipole moment. At a coarse-grained scale larger than that of the individual grains, the piezoelectric coefficients which couple the electrical and the elastic properties are proportional to the local polarization. Due to the large heterogeneity of the ceramic, large spatial inhomogeneities are present in the local dielectric constant. As a result, under a macroscopic applied electric field, the polarization will exhibit large spatial fluctuations. In reference [8], it has been found that the distribution of local field displays in general a double-peak character with a variance linearly correlated to the deviation of the mean polarization from the Lorentz value. This implies that the piezoelectric coefficient is not uniform in the ceramic but fluctuates spatially across the sample.

Piezoelectricity is quantified by the piezoelectric tensor relating strain and electric field. This tensor is deeply related to the symmetry of the system. Three crystal classes contain most of the ferroelectric compounds which have proved important in the field of piezoelectric ceramics : tetragonal, rhombohedral and orthorhombic. However, a piezoelectric ceramic has only one type of piezoelectric matrix regardless of the symmetry of the constituant crystals since the ceramic is initially isotropic and the isotropy is destroyed in the direction of the poling field. Of course, isotropy is maintained in the plane at right angle to it. By analogy with single crystals, the symmetry of an initially random ceramic which has been poled to give a piezoelectric body can be termed $\infty \mathrm{mm}$ (cylindrical polar symmetry) [5].

The general piezoelectric equations relating the electric and elastic variable can be written in the general form

$$
\begin{aligned}
\varepsilon & =P_{\varepsilon \sigma} \sigma+P_{\varepsilon E} E \\
D & =P_{D \sigma} \sigma+P_{D E} E
\end{aligned}
$$

where $\varepsilon$ and $\sigma$ are the strain and stress second order tensors and $D$ and $E$ are the electric displacement and electric field respectively $P_{\varepsilon \sigma}^{-1}$ is the usual Hooke elastic tensor relating stress to strain and it may depend on electrical properties. $P_{D E}$ is the usual dielectric tensor relating the electric field to the electric displacement. The piezoelectric tensors $P_{D \sigma}$ and $P_{\varepsilon E}$ describe the coupling between electrical and mechanical behaviour. The reciprocity of the piezoelectric effect is reflected in the symmetry $P_{\varepsilon E}=P_{D \sigma}$ [5]. For a poled ceramic, the tensor $P_{\varepsilon E}$ can be represented in the form [5] 


\begin{tabular}{|c|c|c|c|c|c|c|}
\hline & $\sigma_{11}$ & $\sigma_{22}$ & $\sigma_{33}$ & $\sigma_{23}$ & $\sigma_{31}$ & $\sigma_{12}$ \\
\hline$D_{1}$ & 0 & 0 & 0 & $P_{14}$ & $P_{15}$ & 0 \\
\hline$D_{2}$ & 0 & 0 & 0 & $P_{24}=P_{15}$ & $-P_{14}$ & 0 \\
\hline$D_{3}$ & $P_{31}$ & $P_{32}=P_{31}$ & $P_{33}$ & 0 & 0 & 0 \\
\hline
\end{tabular}

with only four independent non-vanishing piezoelectric coefficents $P_{31}, P_{33}, P_{14}$ and $P_{15}$. The subscript 3 refers to the poling axis and 1 and 2 refer to arbitrarily chosen orthogonal axes in the plane normal to 3 . Subscripts 4 and 5 refer to shear stress and strain in planes normal to the 1 and 2 axes respectively. The planar isotropy of poled ceramics is expressed in their piezoelectric coefficient by the equalities $P_{24}=P_{15}$ and $P_{32}=P_{31}$.

One is often interested in the hydrostatic behaviour of the composite which may constitute a major application [4]. In this case, since $\sigma_{11}=\sigma_{22}=\sigma_{33}=$ pressure, the relevant piezoelectric coupling coefficient is

$$
P_{\mathrm{H}}=P_{31}+P_{32}+P_{33}=2 P_{31}+P_{33} \text {. }
$$

In non-porous ceramics, it is experimentally often observed that $P_{31} \approx-P_{33} / 2$ which leads to a very small $P_{\mathrm{H}}$. We will see that in the porous case $P_{\mathrm{H}}$ may become significant due to the large decrease of $P_{31}$.

2.2 GEOMETRY OF THE PERCOLATION MODEL. - We focus on the limiting case of a strong heterogeneity where percolation ideas may apply and lead to simple and universal predictions. In this case, the simplicity of the analysis comes from the clear separation of three scales, the microscopic grain size $R$, the percolation mesh size $\xi$ which diverges as the percolation threshold is approached and the macroscopic size $L$ of the system $(R \ll \xi \ll L)$. To fix ideas, consider the case of a piezoelectric ceramic made of sintered spherical grains of radius $R$. The grains occupy a fraction $p$ of space and the remaining $(1-p)$ fraction is either void or is a non-piezoelectric matrix with very large compliance, low dielectric constant ( $\varepsilon_{\mathrm{r}} \approx 1$ such as for voids) and zero piezoelectric coefficient. As $p$ decreases from 1 , the ceramic becomes more and more porous until it becomes macroscopically disconnected at the percolation threshold $0<p_{c}<1$. In practice, porous ceramics with a porosity as high as $50 \%$ can be made. With an improved method of preparation, higher porosity could be reached with $p$ nearer to $p_{c}$ (see e.g. Ref. [9] where a porosity larger than $90 \%$ can be reached).

As $p$ approaches $p_{c}$, the percolating grain system can be seen as a single percolating cluster (finite clusters are not considered). If the embedding medium has a much smaller dielectric constant than the material itself, only a small subpart of this structure has an active role in the transport properties. It is the "backbone », which can be approximately depicted using the «nodes-links-and-blobs » scheme [10]: Quasi-one-dimensional string segments (« macrolinks ») tie together a set of macro-nodes whose typical separation is the percolation length $\xi$ :

$$
\xi \propto\left(p-p_{c}\right)^{-\nu}
$$

with $\nu \approx 0.89$ in $3 \mathrm{~d}$ and $\nu=4 / 3$ in $2 \mathrm{~d}$ [1]. Each string consists of several sequences of « singly connected » bonds of total number

$$
L_{1} \approx\left(p-p_{\mathrm{c}}\right)^{-\zeta}
$$


with $\zeta=1$ [11] in series with thicker regions or «blobs ». Furthermore, branched structures decorate this «backbone » and form « dangling » or « dead » arms. For transport properties such as the electrical conductivity and the mechanical moduli, only the backbone carries a current, a polarization or a force and is therefore relevant. In summary, as $p$ approaches $p_{\mathrm{c}}$ the complex system can be modelled as a homogeneous macro-lattice of typical mesh size $\xi$. Note that as the material becomes very porous, its compliance increases dramatically : a finite applied stress creates a very large strain resulting in a diverging local polarization of the backbone. Such a concentration effect acting on forces and torques which are transmitted by the percolating backbone may not appear for the electrical properties since electric fields are supported by vacuum. We will recover the concentration effect in the limit where the dielectric constant $\varepsilon_{\mathrm{r}}$ of the backbone is much larger than one. In this limit, we may suppose that the electric displacement field $D=\varepsilon_{0} \varepsilon_{\mathrm{r}} E$ is essentially carried in regions of space with the large $\varepsilon_{\mathrm{r}}$.

Following the derivation of the macroscopic mechanical properties in the critical region [1], the macroscopic piezoelectric properties are obtained once the piezoelectric characteristics of each macro-link of size $\xi$ are known [12]. To reach this goal, one has

1 ) to identify the relevant modes of deformation at the microscopic $R$-scale which control that at the $\xi$-scale, and

2 ) to use the correct association rules in order to go from the $R$-scale to the $\xi$-scale.

2.3 ELASTIC VERSUS ELECTRIC TRANSPORT. - The electrical conductivity, $\Sigma$, of a percolation system consisting of conductors (proportion $p$ ) and insulators (proportion $1-p$ ) vanishes at the percolation threshold. It can be characterized by a critical index $t$ such that :

$$
\Sigma \propto\left(p-p_{\mathrm{c}}\right)^{t}
$$

for an infinite size system. $t$ has been numerically estimated to be 1.300 in two dimensions [13], and 2.0 in three dimensions. To take into account the finite size, $L$, of the medium, one introduces a scaling function $\varphi$ such that

$$
\Sigma \propto\left(p-p_{\mathrm{c}}\right)^{t} \varphi(L / \xi)
$$

Usual scaling arguments leads naturally to the asymptotic properties of this function $\varphi$ :

$$
\begin{array}{ll}
\varphi(x) \propto x^{-t / \nu} & \text { for } \quad x \ll 1 \\
\varphi(x) \propto x^{0} & \text { for } x \gg 1 .
\end{array}
$$

In the following, we will present an approximate computation of the exponent $t$ (and others), using the previously introduced node-link-and-blob scheme. It provides a rough estimate of $t$ as

$$
t=\zeta+\nu(d-2)
$$

Although the numerical value of this estimate is somewhat different from the true « value » in two dimensions (1 versus 1.3 ), it gives better results for higher space dimensions. Moreover, the estimate $\zeta+\nu(d-2)$ can be shown to be a lower bound on this exponent [1]. This bound can be supplemented by an upper bound constructed in a very similar way, using the minimal path instead of the set of singly connected bonds. We will not repeat in the following the argument used to construct the second bound, however, it can be easily obtained by formally 
replacing the exponent $\zeta$ by $\nu D_{\min }$, where $D_{\min }$ is the fractal dimension of the minimal path, i.e. 1.130 in two dimensions, and 1.35 in three dimensions [14].

As mentioned in the introduction, the critical behavior of the elastic modulus is different from that of the conductivity. For an infinite system, the elastic modulus $E$ scales as

$$
E \propto\left(p-p_{\mathrm{c}}\right)^{\tau}
$$

$\tau$ has been argued to amount to $t+2 \nu$ [12]. Numerically, in two dimensions, this conjecture seems fulfilled, $\tau=3.96 \pm 0.04$ [15]. In three dimensions, we expect $\tau \approx 3.8$. In the following derivation of the behavior of the piezoelectric tensor at percolation threshold, we will have the opportunity to explain the origin of the difference between $t$ and $\tau$.

Note that although the exponent $\zeta$ is found to be exactly equal to 1 in any dimensions [11], we keep it under its symbolic notation in the following discussion. This will allow us to identify the transport exponents for the conductivity and the elastic modulus from their dependence as a function of $\zeta$ and replace their approximate expressions by their precise numerically known values.

\section{Bound-type estimate of the critical piezo-electricity.}

3.1 CASE OF $\varepsilon_{\mathrm{r}} \rightarrow+\infty$. - Let us consider the ideal case of a piezoelectric ceramic above the percolation threshold $p_{\mathrm{c}}$ with a very large dielectric constant $\varepsilon_{\mathrm{r}}$ imbedded in a matrix with zero elastic modulus and permittivity one. Consider a macro-link consisting in $L_{1} \sim \xi^{\zeta / \nu}$ piezoelectric singly-connected micro-bonds characterized by the simplified piezoelectric equations

$$
\left[\begin{array}{l}
x \\
v
\end{array}\right]=\left[\begin{array}{cc}
s & c \\
-c & a
\end{array}\right]\left[\begin{array}{l}
f \\
q
\end{array}\right]
$$

where $x$ is the displacement of the microbond, $f$ the force exerted on it, $s$ its compliance, $c$ its piezoelectric coefficient, $v$ the voltage drop across it, $q$ its charge and $a^{-1}$ its capacity.

We can argue that the total displacement of the macro-link is the sum over the $L_{1} \sim \xi^{\zeta / \nu}$ micro-bonds of the displacement of pure elastic origin plus that stemming from the piezoelectric coupling

$$
x_{\xi}=x_{1}+x_{2}
$$

with

$$
x_{1} \propto s f \xi^{(\zeta / \nu+2)} .
$$

The exponent $(\zeta / \nu+2)$ stems from the fact that if a compressional stress is applied at the macroscopic scale $L$, the dominant deformation mode at the scale $\xi$ is flexion. This means that most of the elastic energy is stored under the form of flexure deformation energy. This is due to the role of lever effects of long arms (of typical length $\xi$ ) which reinforce the role of flexion with respect to the other deformation modes [12]. More quantitatively, if the typical force acting on a macro bond is $f$, then there are also torques $\mathbf{M}$ of the order of $f \xi$. The dominant mode of deformation of the macrobond will be flexural. We can relate the rotation $\theta$ between both ends of the bond to the torque by a flexural modulus which scales as the conductance : $\theta \propto \xi^{\zeta / \nu}(f . \xi)$. Finally we conclude by expressing the average displacement $x_{1}$ as proportional to $\theta$. $\xi$. Therefore : $x_{1} \propto \xi^{\zeta / \nu+2} f$. The factor $\xi^{2}$ which distinguishes the conductivity from the mechanical behaviour comes from two contributions :

1) the action of torques with lever of arms of size $\xi$ and

2) the effect of rotations with lever of arm also of size $\xi$. 
In order to estimate the node displacement induced by the effect of an applied charge $q$, we note that rotations are now irrelevant since the charge is a scalar quantity. The only contribution with non-zero average comes from a direct coupling from the electric charges to local contractions or extensions. Assuming that the charges $q$ are uniformly distributed along the macro-bond, we find readily a homothetic relationship

$$
x_{2} \propto c \xi q .
$$

Due to the scalar proportionality between local deformation and local charge, only the end to end distance $\xi$ controls the global macro-link deformation.

In the same way, the voltage drop over the macro-link is the sum

$$
v_{\xi}=v_{1}=v_{2}
$$

of the voltage of pure electric origin

$$
v_{1} \sim a \xi^{\zeta / \nu} q
$$

plus that created by the piezoelectric coupling

$$
v_{2}=-\sum_{i} c \mathbf{f} \cdot \mathbf{u}_{i}=-c \mathbf{f} \cdot \boldsymbol{\xi}
$$

where $\mathbf{f}$ is the characteristic compressional force acting at the microscale. Expression (15) corresponds to the association in series of a number of the order of $\xi^{\zeta / \nu}$ different capacitors, each one corresponding to a single micro-bond. From equation (16), we deduce that

$$
v_{2} \propto-c f \xi .
$$

These expressions are valid only when the blobs which decorate the macro-link are neglected. Contrarily to the usual case, this does not lead directly to lower bounds for the critical exponents since the local tensor considered is not a quadratic positive form. However, a more careful analysis using admissible fields allows one to recover the usual bounds for the two diagonal terms of the piezoelectric tensor, namely the elastic modulus and the dielectric constant.

At a macroscopic level, one applies an homogeneous stress $\sigma$ and an electric displacement $D$ which are related to $f$ and $q$ by

$$
\begin{aligned}
& f \sim \sigma \xi^{d-1} \\
& q \sim D \xi^{d-1} .
\end{aligned}
$$

Equation (18) writes that the stress is supported only by the percolation backbone. Equation (19) writes that the electric displacement field is essentially concentrated on the backbone. This assumption becomes correct in the limit of large $\varepsilon_{\mathrm{r}}$.

The corresponding macroscopic electric field and elastic deformation $\varepsilon$ are given by $\varepsilon=x / \xi$ and $E=v / \xi$ which yields

$$
\left[\begin{array}{l}
\varepsilon \\
E
\end{array}\right] \propto\left[\begin{array}{cc}
\xi^{\zeta / \nu+d} & \xi^{d-1} \\
-\xi^{d-1} & \xi^{\zeta / \nu+d-2}
\end{array}\right]\left[\begin{array}{l}
\sigma \\
D
\end{array}\right] .
$$

In order to obtain a symmetrical positive quadratic form for the energy, we write $(\varepsilon, D)$ as a function of $(\sigma, E)$ : 


$$
\left[\begin{array}{c}
\varepsilon \\
D
\end{array}\right] \propto\left[\begin{array}{ll}
\left(p-p_{\mathrm{c}}\right)^{-\tau} & \left(p-p_{\mathrm{c}}\right)^{t-\nu(d-1)} \\
\left(p-p_{\mathrm{c}}\right)^{t-\nu(d-1)} & \left(p-p_{\mathrm{c}}\right)^{t}
\end{array}\right]\left[\begin{array}{l}
\sigma \\
E
\end{array}\right]
$$

We have replaced the lower bound estimates $\zeta+(d-2) \nu$ by the exponent $t$ and $\zeta+d \nu$ by $\tau$. We thus recover the scaling of the compliance which diverges as $\left(p-p_{\mathrm{c}}\right)^{-\tau}$, that of the dielectric constant which vanishes as the dilution approaches $p_{\mathrm{c}}$ as $\varepsilon_{\mathrm{r}}(p) \propto$ $\varepsilon_{\mathrm{r}}\left(p-p_{\mathrm{c}}\right)^{t}$. Note that the matrix is symmetric in agreement with the reciprocity of the direct and converse effects which, following Onsager, must hold whatever the value of the dilution. The validity of the Onsager reciprocity relation can be traced back in this case to the similarity of argument used to obtained $x_{2}$ and $v_{2}$ (Eqs. (13) and (17)).

From equation (21), we obtain that the piezoelectric coupling coefficient defined by equations $(1,2)$ scales as

$$
P_{33}^{\text {percolation }}=\left(\frac{\partial D}{\partial \sigma}\right)_{E}=\left(\frac{\partial \varepsilon}{\partial E}\right)_{\sigma} \propto\left(p-p_{\mathrm{c}}\right)^{\theta}
$$

with an exponent $\theta$ whose estimate is $\theta=t-\nu(\bar{d}-1)$ or numerically $\theta \approx-0.03$ in two dimensions and $\theta \approx-0.2$ in three dimensions. Let us note that, in this case, it is not possible to obtain a direct bound on $\theta$. In view of the small value of $\theta$ obtained within our scaling theory and the uncertainty of its estimation, we can conclude that the spatial average of the macroscopic piezoelectric coefficient is essentially non-critical in practice although the mechanical and electrical behaviours exhibit their usual scaling. More precisely, it will be very difficult to determine experimentally or numerically whether $\theta$ is indeed vanishing or if it has a small value. However, the theoretical question of the precise value taken by $\theta$ remains interesting but unsettled.

Note that the critical behaviour of the macroscopic dielectric constant and of the piezoelectric coupling coefficient should cross-over to a finite regime since in practice, even if $\varepsilon_{\mathrm{r}}$ is very large (a few thousand), it is still finite. This can be analysed with the tools of [16].

3.2 CASE OF $\varepsilon_{\mathrm{r}} \approx 1$. - In this case, the electrical properties of the system are no longer critical in contrast to the elastic ones will remain critical at the percolation threshold. The difference with the previous limit $\varepsilon_{\mathrm{r}} \rightarrow+\infty$ stems from the fact that the electric field invades all the space and is no longer confined on the piezoelectric percolating medium. This implies that the strong electrical screening effects appearing in the case $\left(\varepsilon_{\mathrm{r}} \rightarrow+\infty\right)$ and which allowed a local analysis to be developed are no longer at work in the finite $\varepsilon_{\mathrm{r}}$ case. In principle, one can expect electrical long. range interactions which may change the nature of the problem completely. In particular, the piezoelectrical coupling will induce a strengthening of the material, which a priori may even modify the way the elastic modulus vanishes at $p_{\mathrm{c}}$. In the sequel, we present scaling arguments which suggest that these long range dipolar interactions do not change the leading critical behaviour of the elastic and piezoelectric coefficients.

We assume that the piezoelectric material can be characterized locally as previously by a matrix of the form given by equation (10) whereas the embedding material has no mechanical strength, nor piezoelectric coupling; thus its behavior can be written symbolically as :

$$
\left[\begin{array}{l}
x \\
v
\end{array}\right]=\left[\begin{array}{cc}
\infty & 0 \\
0 & a
\end{array}\right]\left[\begin{array}{l}
f \\
q
\end{array}\right]
$$

As previously, we consider a cube of size $\xi$, subjected to a force

$$
f=\sigma \xi^{d-1}
$$


and with an electric charge

$$
q=D \xi^{d-1}
$$

Equation (25) stems from the equation of continuity on the electric displacement field $D$ at a surface of $\varepsilon_{r}$-discontinuity : a surface density charge proportional to the discontinuity in $D$ appears.

All the force will be transmitted by the piezoelectric material, whereas the electric field will get contributions from both materials, thus we call $q_{1}$ and $q_{2}$ respectively the typical charges on each piezoelectric bond and on each soft bond. Therefore $q$ is written

$$
q=q_{1}+q_{2}(\xi / R)^{d-1}
$$

where $R$ is the typical size of the bonds or grains. Equation (26) shows that typically only a single piezoelectric bond crosses the surface of the cube whereas matrix bonds of the order of $(\xi / R)^{d-1}$ cross the cube surface (see Fig. 1$) . q_{2}$ can be obtained from the fact that the medium has no critical geometry. It should thus be proportional to the potential difference $v / \xi$ accross one bond :

$$
q_{2} \propto(1 / a) v / \xi
$$

where $v$ is the potential drop over a macro-bond of length $\xi$. The elastic displacement can be again decomposed into two main contributions, the first one $x_{1}$ comes from a direct elastic deformation, neglecting all coupling. The second term, on the contrary, takes into account the fact that the electric field in the medium will induce an elastic displacement, $x_{2}$. We thus have

$$
x_{1} \propto s \xi^{\zeta / \nu+2} f
$$

and

$$
x_{2} \propto c q_{1} \xi
$$

which, by using equations (26) and (27) gives

$$
x_{2} \propto c \xi\left(q-(1 / a)(v / \xi)(\xi / R)^{d-1}\right) .
$$

Thus

$$
x \propto s \xi^{\zeta / \nu+2} f+c \xi\left(q-(1 / a)(v / \xi)(\xi / R)^{d-1}\right) .
$$
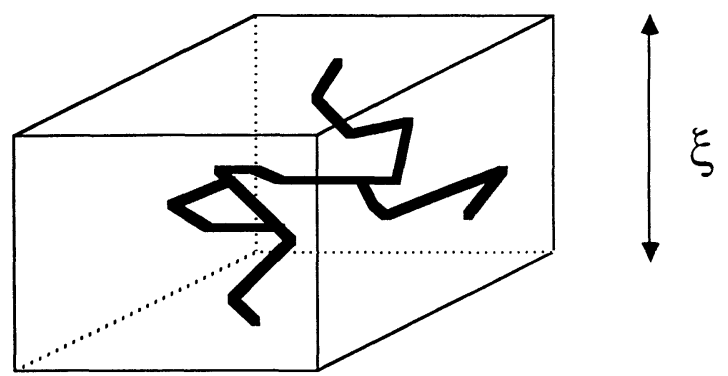

Fig. 1. - Schematic representation of a typical cell of size $\xi$ containing a single piezoelectric macrobond which crosses the cube and connects its lower and upper surface. 
The factor $\xi$ can be seen as the result of a uniform expansion of the medium on a scale $\xi$. Similarly the potential difference $v$, when considered along the piezoelectric material, also gives two contributions :

$$
v \propto-c f \xi+a \xi\left(q-(1 / a)(v / \xi)(\xi / R)^{d-1}\right) .
$$

Expressing $v$ and $x$ as a function of $f$ and $q$, and using the electric field $E=v / \xi$ and the deformation $\varepsilon=x / \xi$, we finally obtain :

$$
\begin{aligned}
\varepsilon & =\left\{s \xi^{\zeta / \nu+d}+\left(c^{2} / a\right) \xi^{d-1}\right\} \sigma+c D \\
E & =-c \sigma+a D
\end{aligned}
$$

or

$$
\left[\begin{array}{c}
\varepsilon \\
D
\end{array}\right] \propto\left[\begin{array}{ll}
\left(p-p_{\mathrm{c}}\right)^{-\tau} & \left(p-p_{\mathrm{c}}\right)^{0} \\
\left(p-p_{\mathrm{c}}\right)^{0} & \left(p-p_{\mathrm{c}}\right)^{0}
\end{array}\right]\left[\begin{array}{l}
\sigma \\
E
\end{array}\right]
$$

where we have identified the exponent $\tau=\zeta+d \nu$. We see that the strengthening of the medium by the piezoelectric coupling is not dominant compared to the direct elasticity. We obtain finally that in this expression, the only critical term is the elastic stiffness that will go to zero as if no coupling would exist, (as $\left.\left(p-p_{c}\right)^{\tau}\right)$. The coupling $c$ does not exhibit any critical behavior. However, this is a simple consequence of the choice of variables. In fact, upon inversion, equation (32) yields

$$
\left[\begin{array}{l}
\sigma \\
E
\end{array}\right] \propto\left[\begin{array}{cc}
\left(p-p_{\mathrm{c}}\right)^{\tau} & -\left(p-p_{\mathrm{c}}\right)^{\tau} \\
-\left(p-p_{\mathrm{c}}\right)^{\tau} & \left(p-p_{\mathrm{c}}\right)^{0}
\end{array}\right]\left[\begin{array}{c}
\varepsilon \\
D
\end{array}\right]
$$

Therefore, one sees that a very crude argument obtained by setting formally the exponent $t$ to zero in the previous case $\left(\varepsilon_{\mathrm{r}}=\infty\right)$, gives the correct scaling for this case too. This result was not expected beforehand because of the complex interplay of the critical elastic behaviour and the non-critical electric one; however, these subtleties seem to be irrelevant for the leading critical behaviour.

\section{Numerical results on the beam model in two dimensions.}

In the beam model [7] (see Fig. 2), which contains full bond-bending elasticity, there are three variables on each site: the coordinates $x_{i}$ and $y_{i}$ of its displacement and an angle

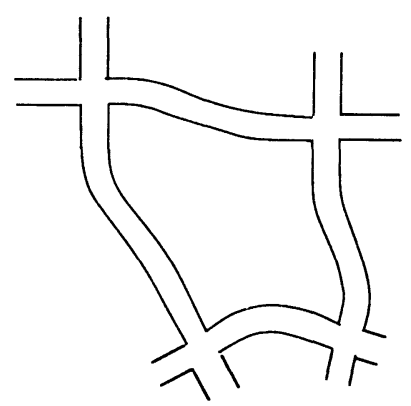

Fig. 2. - Schematic representation of the beam model in two dimensions. The bonds are elastic beams soldered at the two ends in such a way that they must enter each site tangentially, therefore insuring that the deformation is localized solely on the beams. 
$\theta_{i}$. The bonds are elastic beams soldered at the two ends in such a way that they must enter site $i$ tangentially under the angle $\theta_{i}$ (measured with respect to the undistorted square lattice). So as to deal with homogeneous variables, we call $z$ the rotation $\theta$ multiplied by the length of a bond. In order to describe the piezoelectric coupling, we assume furthermore the existence of a fourth variable, namely the voltage $v_{i}$ at each site. The energy of the beam is the sum of the elastic, electric and piezoelectric energy. The elastic energy is the sum of elongation, shear and flexural energy. The electric energy is quadratic in the voltage. The piezoelectric energy contains cross-terms involving displacement and electric voltage. The expression relating the generalized displacements to the generalized forces on each site is written, for a bond along the $x$ axis :

$$
\left[\begin{array}{c}
F_{x} \\
F_{y} \\
M \\
q
\end{array}\right]=R\left[\begin{array}{c}
x \\
y \\
z \\
v
\end{array}\right]=\left[\begin{array}{cccc}
d & 0 & 0 & -e / 2 \\
0 & a & b & 0 \\
0 & b & c & 0 \\
e / 2 & 0 & 0 & f
\end{array}\right]\left[\begin{array}{l}
x \\
y \\
z \\
v
\end{array}\right]
$$

if we assume that all four variables $(x, y, z, v)$ are zero at one end of the beam. $F$ is a force, $M$ is a torque divided by the length of the bond. $a, b, c$ and $d$ are elastic constants, $f$ is a dielectric constant, and finally $e$ is a piezoelectric coupling constant. Only three of the four elastic constants are free parameters. One of them can be expressed as a function of the others, by writing the rotational invariance of the system.

This matrix $R$ is the most general form which respects the symmetries of the system : The symmetry of the beam along its axis decouples the $x$ and $v$ coordinates from the other variables $y$ and $z$. The top left $3 \times 3$ submatrix of $R$, is nothing but the usual elastic rigidity tensor of a beam. It defines a quadratic energy that is invariant under translations and infinitesimal rotations. The opposite signs of $R_{14}$ and $R_{41}$ implies that the matrix relating $\left(F_{x}, F_{y}, M, v\right)$ to $(x, y, z, q)$ is positive definite. The corresponding matrix for a bond along the $y$ direction can be obtained easily by considering a rotation of $\pi / 2$.

With this definition of energy, it is straighforward to generalise the transfer-matrix algorithm used for instance in reference [17] to compute the conductivity of percolation lattices. This algorithm is known to give the most precise estimates of various transport critical exponents of percolation, by exploiting finite-size effects. We construct a very long strip (typically $5 \times 10^{4}$ or $10^{5}$ bonds) of width $w$ (varying in our case from 2 to 40 bonds) made out of a lattice exactly at the percolation threshold, $p_{\mathrm{c}}$. The long edges of the strip are connected to two infinitely rigid bars, one of which is fixed, while the other can be attributed any set of parameters $\left(x^{*}, y^{*}, z^{*}, v^{*}\right)$. For those strips, we compute exactly the corresponding matrix $R$ for the variables $\left(x^{*}, y^{*}, z^{*}, v^{*}\right)$. The length of the strip is chosen to be long enough so that $R$ converges toward a well-defined matrix. We then study the evolution of the coefficient of this matrix as a function of the width of the strip, $w$. From the general theory of finite size scaling, we expect any coefficient $R_{i j}$ to follow a power-law with $w$, with an exponent $\alpha / \nu$, where $\nu$ is the percolation correlation exponent, such that

$$
R_{i j} \propto w^{\alpha / \nu} \text { at } p=p_{\mathrm{c}}
$$

if

$$
R_{i j} \propto\left(p-p_{\mathrm{c}}\right)^{-\alpha} \text { in an infinite system. }
$$

A very careful analysis of the elastic critical behavior of percolation systems using the transfer-matrix algorithm on lattices different than ours [15] has revealed that the critical regime (strictly speaking only valid for large widths) was affected for width up to 100 by very 
strong corrections terms, that renders very difficult the estimate of the asymptotic critical exponent. We therefore expect this drawback to be even stronger in our case, since we add another coupling to the elastic behavior. We will thus present our results as rough estimates, since the maximum width $(w=40)$ we considered does not allow us to take into account corrections to scaling. However, the system sizes considered (length $10^{5}$ for $2 \leqslant w \leqslant 28$ and $5 \times 10^{4}$ for $30 \leqslant w \leqslant 40$ ), represent a non-negligible numerical effort (more than $100 \mathrm{CPU}$ hours on an IBM 3090 computer).

We only studied the first situation explicited in this article, namely the case where $\varepsilon_{\mathrm{r}}$ tends to infinity. Table I gives the value of the exponents obtained from the numerical simulations, compared with the theoretical estimates extracted from section 3.1 upon inversion of the matrix appearing in equation (20), since in these simulations, $\varepsilon$ and $E$ were the control parameters. Figure 3 shows the corresponding data from which the exponents are extracted.

Table I. - Comparison between the numerical estimates obtained in the transfer-matrix study of this problem and the theoretical expectation from section 3.1. The «Co. " and «Sh. " stand for compression and shear respectively. From equation $(21)$, we expect $(\partial \sigma / \partial E)_{\varepsilon} \sim\left(p-p_{c}\right)^{\alpha}$ with $\alpha=\tau+t-\nu(d-1)$. Since $\theta=t-\nu(d-1) \approx-0.03$ in two dimensions, we predict an exponent $\alpha / \nu$ very close to $\tau / \nu$. This is agreement with the similar apparent exponents found in our numerical simulations for these two cases. Note that our numerical estimate $t / \nu=0.95$ is rather good when compared to the convergence of the mechanical and piezoelectric exponents but not much so when compared to the usual accuracy [17] expected within the transfer matrix approach for strips of size $w \approx 20-40$. This is due to the coupling with the mechanical behaviour induced by the piezoelectric effect which endorses the conductivity exponent with large corrections to scaling as occurs for the mechanical exponent.

\begin{tabular}{|l|c|c|c|}
\hline & Best numerical value & $\begin{array}{c}\text { Numerical value } \\
\text { from this work }\end{array}$ & Our theoretical estimate \\
\hline$t / \nu$ & $0.9745[13]$ & 0.95 & $t / \nu=0.97$ \\
\hline$\tau / \nu($ Co. $)$ & $2.97[15]$ & 2.0 & $\tau / \nu=2.97$ \\
\hline$\tau / \nu($ Sh. $)$ & $2.97[15]$ & 2.0 & $\alpha / \nu=2.97$ \\
\hline$(\partial \sigma / \partial E)_{\varepsilon}($ Co. $)$ & - & 2.0 & $\alpha / \nu=2 t / \nu+3-d=2.95$ \\
\hline$(\partial \sigma / \partial E)_{\varepsilon}(\mathrm{Sh})$. & - & 2.0 & $\alpha / \nu=2 t / \nu+3-d=2.95$ \\
\hline
\end{tabular}

From table I, we note that although the first obtained exponent compares well with the expectation, all other numerical results are well below. This fact should be compared to the case of the elastic modulus [15] where huge corrections to scaling were obtained for small-size systems. In particular, we can extract from reference [15] the "apparent » value of the exponent $\tau / \nu$ for a width $w=30: 《 \tau / \nu » \approx 2.2$ which is consistent with our data. More importantly, we also note that the approximate equality of the four last exponents that we expect from section 3.1 is verified within numerical accuracy. It can also be seen from the apparent parallelism of the data points presented in figure 3. The data obtained in our simulations thus comfort our theoretical prediction. Our expectation that the critical 


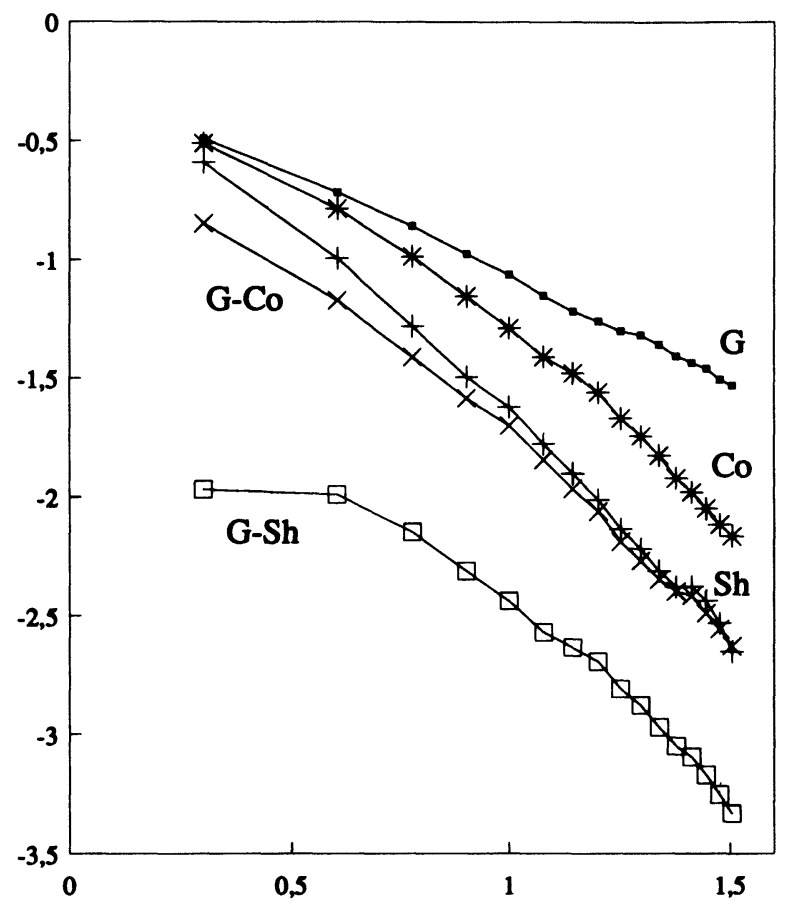

Fig. 3. - Log-log plot of the conductivity $(G)$, the elastic modulus for compression (Co) and shear (Sh) and the piezoelectric coupling constant for compression $(G-\mathrm{Co})$ and shear $(G-S h)$ as a function of the strip width. The slopes of the curves give the exponents reported in table I.

exponent $\alpha / \nu=2 t / \nu+3-d \approx 2.95$ of the piezoelectric coupling coefficients $(\partial \sigma / \partial E)_{\varepsilon}$ (Co.) and $(\partial \sigma / \partial E)_{\varepsilon}($ Sh.) be equal (with a precision better than $1 \%)$ to $\tau / \nu$ is parallel to our finding (see Eq. (22)) that the critical exponent $\theta$ for $P_{33}$ is nearly vanishing. A definite difference between $\alpha / \nu$ and $\tau / \nu$ would imply a non-vanishing value for $\theta$. Unfortunately, our numerical simulation do not have a sufficient precision to settle this point. They however confirm our theoretical prediction that $\theta$ is small.

Note that our beam model does not include a microscopic piezoelectric coupling under shear. However, a macroscopic one appears naturally. This stems from the fact that, due to percolation disorder, a macroscopic shear will introduce local extensions and compressions along the microscopic beams which will couple the electrical and mechanical behaviours thus leading to an apparent macroscopic piezoelectric shear piezoelectric coefficient.

The piezoelectric effect under a shear stress is described by the coupling coefficient $(\partial \sigma / \partial E)_{\varepsilon}($ Sh. $)$ which is found to behave similarly to the compressional coupling coefficient. In fact, it can be analysed in a similar fashion as presented for the compression coupling coefficient and should also exhibit the same critical behaviour (as verified numerically in Fig. 3) due to the existence of the stress enhancement effect. However, as seen in figure 3, its modulus is at least one order of magnitude smaller than that of the compression coupling coefficient. This can be rationalized from the requirement that, for reason of in-plane rotational symmetry, $(\partial \sigma / \partial E)_{\varepsilon}($ Sh. $)$ has to vanish again in the infinite size limit. Due to finite size effects, $(\partial \sigma / \partial E)_{\varepsilon}($ Sh. $)$ is however not strictly zero but remains much smaller than other coefficients. 


\section{Discussion.}

Let us conclude by the following remarks.

1) Our work has been concerned with the critical behaviour of the longitudinal piezoelectric coupling coefficient $\mathrm{P}_{33}$ near the percolation threshold. Our principal result is that this macroscopic piezoelectric coefficient is essentially non-critical although the mechanical and electrical behaviours exhibit their usual scaling. This conclusion is forced upon us by the small value of the critical exponent $\theta$ obtained within our scaling theory which is of the order of, or even less than, the uncertainty of its estimation within such an approach. Furthermore, our numerical simulations confirm our theoretical prediction that $\theta$ is small but does not have a sufficient precision to determine whether it could have a small non-vanishing value. We can thus conclude that the spatial average of the macroscopic piezoelectric coefficient is essentially non-critical in practice, in the sense that it will be very difficult to determine experimentally or numerically whether $\theta$ is indeed vanishing or if it has a small value. However, the theoretical determination of the precise value taken by $\theta$ remains an interesting but so far unsettled question.

2) In the simplified beam model used in our numerical computation, we have neglected any transverse piezoelectric coupling. In a real material however, transverse couplings are important and in fact may lead to a very small value of the hydrostatic piezoelectric coupling $P_{\mathrm{H}}$ as seen from equation (3).

Near the percolation threshold, $P_{\mathrm{H}}$ should become large due to a large decrease of $P_{31}$ with respect to $P_{33}$. Indeed, we argue that the macroscopic transverse piezoelectric coupling coefficient $P_{31}^{\text {percolation }}$ and $P_{32}^{\text {percolation }}$ should be non-critical as $p$ approaches $p_{c}$. This is due to the fact that a transverse force induces a vertical deformation which takes a random sign from one site to the other and thus the different contributions should average to a very small amount. This results in a relative enhancement of $P_{\mathrm{H}}$ due to the different behavior of $P_{31}$ and $P_{33}$ (governed by Eq. (22)) as we come closer to the threshold.

3) The value of the efficiency of the mechanical-electrical transduction is given by the socalled « coupling factor » defined by

$$
C=\frac{\text { (mutual energy) }^{2}}{\text { (elastic energy) (electric energy) }} .
$$

In the case of section 3.1, the elastic energy is proportional to $\left(p-p_{\mathrm{c}}\right)^{-\tau} \sigma^{2}$; the electric energy is proportional to $\left(p-p_{\mathrm{c}}\right)^{t} E^{2}$; and finally the mutual energy follows $\left(p-p_{\mathrm{c}}\right)^{t-\nu(d-1)} E \sigma$. This leads to

$$
C \propto\left(p-p_{\mathrm{c}}\right)\{2 t-2 \nu(d-1)\}+(\tau-t)
$$

But, $\tau$ is believed to be exactly related to $t$ by $\tau=t+2 \nu$. This leads to a critical coupling factor in the limit $p \rightarrow p_{\mathrm{c}}$ when the dielectric constant $\varepsilon_{\mathrm{r}}$ of the piezoelectric ceramic is large, with an exponent $2\{t-\nu(d-2)\}$ which amounts to 2.6 in two dimensions and 2.2 in three dimensions. Therefore we conclude that the efficiency of piezoelectric effect vanishes with dilution.

In the case of section 3.2 , a similar computation leads to

$$
C \propto\left(p-p_{\mathrm{c}}\right)^{\tau} .
$$

The mechanical-electrical transduction becomes less and less efficient compared to the mechanical energy which is stored in the backbone. The vanishing of the efficiency is naturally 
stronger than in the previous case : with $\tau / \nu=2.97$ with $\nu=4 / 3$ in two dimensions, this yields $\tau \approx 3.96$.

4) Piezoelectric ceramics are often used as transductors operating at finite frequencies $f=c / \lambda$ where $c$ is the average longitudinal sound velocity and $\lambda$ the corresponding wavelength. As long as $\lambda$ remains larger than $\xi$, our analysis remains valid and our conclusions should apply in this regime. However, at higher frequencies for which $\lambda$ become smaller than $\xi$, the sound wave becomes sensitive to scales smaller than the percolation correlation length $\xi$ at which the structure of the percolating system is self-similar or fractal. For $\lambda<\xi$, the attenuation length of the wave is of the order of its wavelength $\lambda$ : this is the so-called fracton regime [18] for which we expect a vanishing direct and reverse piezoelectric effect.

5) Let us note that in the case of elastic elements free to rotate around their junctions, simple connexity is not a sufficient condition to insure rigidity. In this case, we have a centralforce percolation situation, where the rigidity threshold is much larger than the usual connexity percolation. Therefore, if the bonds are piezoelectric, in a diluted lattice, we should recover exactly the same situation as the one sketched above for $\varepsilon_{\mathrm{r}}=1$, in the proximity of the rigidity threshold. This would result from a recent conjecture [3] which suggests the same elastic and geometric critical properties of central-force percolation as in the case of usual percolation for systems with angular elasticity.

6) We finally note the remarkable following point : we have used in the previous analysis the fact that the typical value of the force $f$, which has to be considered at the scale $\xi$ for the piezoelectric coupling, was just given by a balance equation $f=\sigma^{d-1}$; however due to the existence of torques in the structure, we rather expect the typical value of the local force to be given by $f . \xi / R$, which is much larger. The latter value only gives the order of magnitudes of fluctuations since the balance argument can be shown to give the mean value correctly. This has the practical consequence that the fluctuations of the piezoelectric constant around its mean value will be enormous. We know of course that fluctuations are always large close to the percolation point. However, for this particular quantity, the lever arm effect gives rise to an enhanced fluctuation source which should be of importance and be observed experimentally.

\section{Acknowledgments.}

We are grateful to J. P. Besombes for useful discussions on porous piezoelectric ceramics and E. Guyon for a critical reading of the manuscript.

\section{References}

[1] GuYON E., The physics of random matter in Chance and Matter, Les Houches, session XLVI (Elsevier Science Publisher) 1987.

[2] Feng S. and Sen P. N., Phys. Rev. Lett. 52 (1984) 216 ;

Lemieux M. A., Breton P. and Tremblay A. M. S., J. Phys. Lett. France 46 (1985) L1.

[3] Hansen A. and Roux S., Phys. Rev. B (to appear) ;

Roux S. and HANSEN A., Europhys. Lett. 6 (1988) 301.

[4] Cross L. E., Newnham R. E., Barsch G. R. and Biggers J. V., Piezoelectric and electrostrictive materials for transducers applications, Annual Report, ONR editor, Contract $n^{\circ}$ N00014 (Materials Research Laboratory Edition, The Pennsylvania State University) May 1985. 
[5] Berlincourt D. A., Current D. R. and Jaffe H., Piezoelectric and piezomagnetic materials and their function in transducers in Physical Acoustics (Masson) Vol. 1-A.

[6] Walpole L. J., Elastic behaviour of composite materials : theoretical foundations, Adv. Appl. Mech. 21 (1981) 169.

[7] Roux S. and GuYON E., J. Phys. Lett. France 46 (1985) L999.

[8] Sheng P. and Chen Z., Phys. Rev. Lett. 60 (1988) 227.

[9] Deptuck D., Harrison J. P. and Zawadsky P., Phys. Rev. Lett. 54 (1985) 913.

[10] SKal A. and Shklovski B., Sov. Phys. Semicond. 8 (1976) 1029 ;

DE Gennes P. G., J. Phys. Lett. France 37 (1976) L1.

[11] Coniglio A., Phys. Rev. Lett. 46 (1981) 250.

Pike R. and Stanley H. E., J. Phys. A 14 (1981) L169.

[12] Roux S., J. Phys. A 19 (1985) L463.

[13] Normand J. M., Herrmann H. J. and HajJar M., J. Stat. Phys. 52 (1988) 747.

[14] Herrmann H. J. and Stanley H. E., J. Phys. A 21 (1988) L829.

[15] Zabolitsky G. J., Bergman D. J. and Stauffer D., J. Stat. Phys. 44 (1986) 211.

[16] Straley J. P., Phys. Rev. B 15 (1977) 5733.

[17] Derrida B. and Vannimenus J., J. Phys. A 15 (1982) L557;

Derrida B., Stauffer D., Hermann H. and Vannimenus J., J. Phys. Lett. France 44 (1983) L701.

[18] Alexander S. and Orbach R., J. Phys. Lett. France 43 (1982) L625. 